\title{
GTC/OSIRIS SPECTROSCOPIC IDENTIFICATION OF A FAINT L SUBDWARF IN THE UKIRT INFRARED DEEP SKY SURVEY
}

\author{
N. Lodieu ${ }^{1,2}$, M. R. Zapatero Osorio ${ }^{3}$, E. L. Martín ${ }^{3}$, E. Solano ${ }^{3}$, And M. Aberasturi ${ }^{3}$ \\ ${ }^{1}$ Instituto de Astrofísica de Canarias, C/Vía Láctea s/n, E-38205 La Laguna, Tenerife, Spain; nlodieu@iac.es \\ 2 Departamento de Astrofísica, Universidad de La Laguna, E-38205 La Laguna, Tenerife, Spain \\ ${ }^{3}$ Centro de Astrobiología (CSIC/INTA), 28850 Torrejón de Ardoz, Madrid, Spain \\ Received 2009 November 9; accepted 2009 December 4; published 2009 December 18
}

\begin{abstract}
We present the discovery of an L subdwarf in $234 \mathrm{deg}^{2}$ common to the UK InfraRed Telescope (UKIRT) Infrared Deep Sky Survey Large Area Survey Data Release 2 and the Sloan Digital Sky Survey Data Release 3. This is the fifth L subdwarf announced to date, the first one identified in the UKIRT Infrared Deep Sky Survey, and the faintest known. The blue optical and near-infrared colors of ULAS J135058.86+081506.8 and its overall spectra energy distribution are similar to the known mid-L subdwarfs. Low-resolution optical (700-1000 nm) spectroscopy with the Optical System for Imaging and low Resolution Integrated Spectroscopy spectrograph on the $10.4 \mathrm{~m}$ Gran Telescopio de Canarias reveals that ULAS J135058.86+081506.8 exhibits a strong K I pressure-broadened line at $770 \mathrm{~nm}$ and a red slope longward of $800 \mathrm{~nm}$, features characteristics of L-type dwarfs. From direct comparison with the four known L subdwarfs, we estimate its spectral type to be sdL4-sdL6 and derive a distance in the interval 94-170 pc. We provide a rough estimate of the space density for mid-L subdwarfs of $1.5 \times 10^{-4} \mathrm{pc}^{-3}$.
\end{abstract} Key words: infrared: stars - stars: Population II - subdwarfs - techniques: photometric - techniques: spectroscopic Online-only material: color figure

\section{INTRODUCTION}

Objects cooler than M dwarfs have been classified into two spectral classes: L and T (Martín et al. 1999; Kirkpatrick et al. 1999; Geballe et al. 2002; Burgasser et al. 2006). Hundreds of them are now known as a result of large scale searches of $\mathrm{L}$ and $\mathrm{T}$ dwarf surveys. ${ }^{4}$ However, at lower metallicity, only four L subdwarfs have been announced to date: 2MASS J053253.46+824646.5 (2MASS0532; sdL7; Burgasser et al. 2003), 2MASS J162620.34+392519.0 (2MASS1626; sdL4; Burgasser 2004), 2MASS 061640.06640719.4 (2MASS0616; sdL5; Cushing et al. 2009), and SDSS J125637.13-022452.4 (SDSS1256; sdL3.5; Sivarani et al. 2009; Burgasser et al. 2009). The first three were discovered serendipitously in the Two Micron All Sky Survey (2MASS; Cutri et al. 2003; Skrutskie et al. 2006) archive while the last was found during a search for ultracool subdwarfs in the Second Data Release (DR) of the Sloan Digital Sky Survey (SDSS; York et al. 2000; Abazajian et al. 2005) spectroscopic database. Increasing the number of ultracool subdwarfs is essential to study the chemistry in cool atmospheres with low metal content, the role of metallicity in the shape of the initial mass function (Salpeter 1955; Miller \& Scalo 1979; Scalo 1986), and the impact of chemical composition on the properties of binary stars (Riaz et al. 2008; Jao et al. 2008; Lodieu et al. 2009b).

The UKIRT Deep Infrared Sky Survey (UKIDSS; Lawrence et al. 2007) uses the Wide-Field Camera (WFCAM; Casali et al. 2007) installed on the UK InfraRed Telescope (UKIRT) and the Mauna Kea Observatory (Tokunaga et al. 2002) photometric system described in Hewett et al. (2006). The pipeline processing is described in M. Irwin et al. (2010, in preparation) $)^{5}$ and the WFCAM Science Archive in Hambly et al. (2008). The Large

\footnotetext{
4 See http://dwarfarchives.org for the full list of $\mathrm{L}$ and $\mathrm{T}$ dwarfs.

5 Extensive details on the data reduction of the UKIDSS images are available at http://casu.ast.cam.ac.uk/surveys-projects/wfcam/technical.
}

Area Survey (hereafter LAS), one of the UKIDSS components, will image $\sim 3800 \mathrm{deg}^{2}$ in four filters $(Y J H K$ ) down to $J=$ 19.6 mag with a significant overlap with SDSS, hence providing spectral energy distributions from 0.3 to $2.5 \mu \mathrm{m}$ for millions of sources.

In this Letter, we present the discovery of the first L subdwarf identified in $234 \mathrm{deg}^{2}$ common to the UKIDSS LAS DR2 (Warren et al. 2007) and the SDSS DR3 (Abazajian et al. 2005). In Section 2, we describe the photometric and proper motion selections to identify ultracool subdwarfs in UKIDSS and SDSS. In Section 3, we present the spectroscopic follow-up observations conducted with the Optical System for Imaging and low Resolution Integrated Spectroscopy (OSIRIS) spectrograph mounted on the new $10.4 \mathrm{~m}$ Gran Telescopio de Canarias (GTC) telescope in La Palma (Canary Islands). In Section 4, we discuss the photometric and spectral characteristics of the new object, infer its distance, and compute its tangential velocity. Finally, we conclude with a discussion on the expected number of subdwarfs that could be identified at the completion of UKIDSS.

\section{SAMPLE SELECTION}

We have initiated a photometric and proper motion search for ultracool (spectral types later than M7) subdwarfs by crosscorrelating the UKIDSS and SDSS databases. In the LAS, we have requested only point sources (mergedClass $=-1$ ) and good quality detections (ppErrBits $\leqslant 256$ ). We have employed the following photometric criteria: $Y-J=0.3-0.75 \mathrm{mag}$, $J-K \leqslant 0.7$ mag because of the strong collision-induced $\mathrm{H}_{2}$ absorption beyond $2 \mu \mathrm{m}$ (Linsky 1969), and $z-J \leqslant$ 2.5 mag because the optical-to-infrared colors of subdwarfs are expected to be bluer than their solar metallicity counterparts (Section 4; Figure 1). We have also imposed constraints on the LAS detections, requesting $J=14-18.5$ mag and photometric errors less than 0.2 mag in $Y$ and $J$ and better than 0.25 mag in $H$ and $K$. The bright limit in $J$ was set to place our candidates well above the saturation of the UKIDSS LAS. In addition, we have 


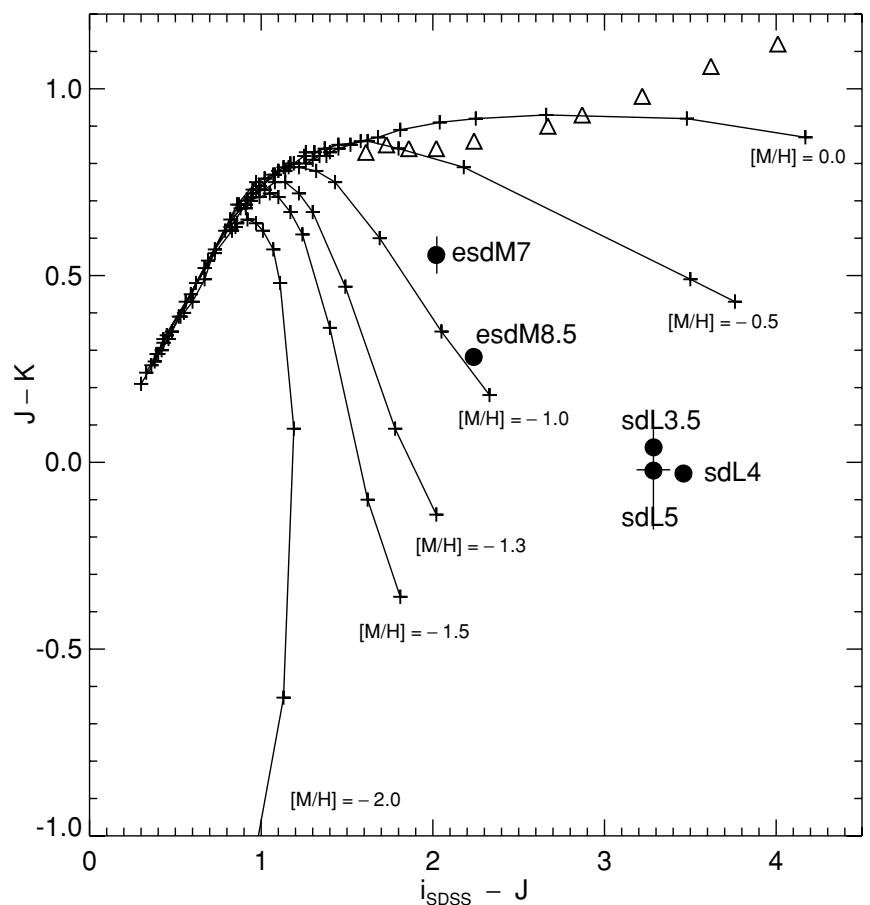

Figure 1. $(i-J, J-K)$ diagram showing the location of two ultracool subdwarfs, SDSS J023557.61+010800.5 (esdM7) and SDSS J020533.75+123824.0 (esdM8.5) from Lépine \& Scholz (2008) and three L subdwarfs as large dots: ULAS1350 (sdL5), 2MASS1626 (sdL4), and SDSS1256 (sdL3.5) with SDSS photometry. Photometric error bars have been added for ULAS1350 and SDSS J023557.61+010800.5; the others being smaller than the size of the symbols Theoretical $10 \mathrm{Gyr}$ tracks for different metallicities are also shown (Baraffe et al. 1997). The colors of M0-M9 dwarfs are overplotted as open triangles (West et al. 2008); L dwarfs lie outside this diagram because of their red $J-K$ colors. A similar version of this plot was used by Scholz et al. (2004) and Burgasser et al. (2009).

requested SDSS detections below the $5 \sigma$ limits in $u(\geqslant 22.3 \mathrm{mag})$ and $g(\geqslant 23.3 \mathrm{mag})$. Besides the photometric criteria, we have imposed a lower limit of $0.18 \operatorname{arcsec}_{\mathrm{yr}^{-1}}$ on the proper motion (Luyten 1980), which is measured as the difference between UKIDSS and SDSS coordinates.

This query returned seven photometric candidates. The photometry of six candidates suggests that they are late-M subdwarfs. Spectroscopic follow-up of these six sources is currently underway. However, one object, ULAS J135058.86+081506.8 (hereafter ULAS1350), is the only source with a negative $J-H$ color and among the faintest ones displaying optical and nearinfrared colors similar to those of 2MASS1626 (sdL4; Burgasser 2004), the second L subdwarf known at the time of our discovery. Table 1 provides the catalogued photometry of ULAS1350 and two other known L subdwarfs as well as a field L5 dwarf for comparison. Therefore, we prioritized this candidate for optical spectroscopy that we finally secured with the GTC in 2009. From the SDSS/UKIDSS cross-match, we inferred a total proper motion of $0.280 \pm 0.025 \operatorname{arcsec}_{\mathrm{yr}^{-1}}$ thanks to the $\sim 3 \mathrm{yr}$ baseline between both observations (2003 April and 2006 July). The typical uncertainty on the difference between the UKIDSS and SDSS astrometry is $0.025 \operatorname{arcsec}_{\mathrm{yr}}{ }^{-1}$ for sources brighter than $J=18.5 \mathrm{mag}$ (see analysis in Dye et al. 2006 and Lodieu et al. 2009a). This proper motion is lower than the motion of the other four L subdwarfs currently known. ULAS1350 is also fainter, suggesting that it lies at a larger distance. Its reduced proper motion $\left(H_{i}=23.45 \mathrm{mag}\right)$ is similar to 2MASS 1626 $\left(H_{i}=23.34 \mathrm{mag}\right)$ and SDSS1256 $\left(H_{i}=23.41 \mathrm{mag}\right)$, supporting its possible low-metallicity status.
Table 1

Photometry and Spectral Type of ULAS J135058.86+081506.8 Along with Two Comparison L Subdwarfs and a Field L Dwarf

\begin{tabular}{lcccc}
\hline \hline & ULAS1350 $^{\mathrm{a}}$ & SDSS1256 $^{\mathrm{b}}$ & 2MASS1626 $^{\mathrm{b}}$ & SDSS1446 $^{\mathrm{a}, \mathrm{c}}$ \\
\hline R.A. & $13: 50: 58.86$ & $12: 56: 37.10$ & $16: 26: 20.34$ & $14: 46: 00.60$ \\
Decl. & $+08: 15: 06.8$ & $-02: 24: 52.5$ & $+39: 25: 19.0$ & $+00: 24: 52.0$ \\
$i-z$ & $1.74 \pm 0.11$ & $1.70 \pm 0.02$ & $1.74 \pm 0.01$ & $2.27 \pm 0.06$ \\
$z-J$ & $1.55 \pm 0.07$ & $1.61 \pm 0.02$ & $1.71 \pm 0.01$ & $2.92 \pm 0.03$ \\
$J-K$ & $-0.02 \pm 0.16$ & $-0.10 \pm 0.02$ & $-0.03 \pm 0.01$ & $1.66 \pm 0.01$ \\
$r$ & $24.30 \pm 0.66$ & $21.80 \pm 0.11$ & $20.61 \pm 0.03$ & $23.19 \pm 0.24$ \\
$i$ & $21.22 \pm 0.09$ & $19.39 \pm 0.02$ & $17.89 \pm 0.01$ & $20.77 \pm 0.05$ \\
$z$ & $19.48 \pm 0.06$ & $17.68 \pm 0.02$ & $16.15 \pm 0.01$ & $18.50 \pm 0.03$ \\
$Y$ & $18.60 \pm 0.05$ & $\ldots$ & $\ldots$ & $16.90 \pm 0.01$ \\
$J$ & $17.94 \pm 0.04$ & $16.16 \pm 0.01$ & $14.43 \pm 0.01$ & $15.58 \pm 0.01$ \\
$H$ & $18.08 \pm 0.10$ & $16.06 \pm 0.01$ & $14.46 \pm 0.01$ & $14.66 \pm 0.01$ \\
$K$ & $17.98 \pm 0.15$ & $16.06 \pm 0.02$ & $14.46 \pm 0.01$ & $13.92 \pm 0.01$ \\
SpT & sdL5 \pm 1.0 & sdL3.5 \pm 0.5 & sdL4 \pm 0.5 & $\mathrm{dL5} \pm 0.5$
\end{tabular}

Notes.

a Optical photometry from SDSS DR7 (AB mag) and near-infrared photometry from UKIDSS LAS DR6 (Vega system).

${ }^{\mathrm{b}}$ Optical photometry from SDSS DR7 (AB mag) and near-infrared photometry from Schilbach et al. (2009).

${ }^{\mathrm{c}}$ Original discovery from Geballe et al. (2002).

\section{SPECTROSCOPIC OBSERVATIONS}

The $10.4 \mathrm{~m}$ GTC started operations in 2009 March at the Observatory del Roque de Los Muchachos (La Palma, Canary Islands). It is currently equipped with one of its Day One instruments, the OSIRIS instrument (Cepa et al. 2000). The OSIRIS spectrograph consists of two $2048 \times 4096$ Marconi CCD42-82 with an 8 arcsec gap between them and operates at optical wavelengths, from 365 to $1000 \mathrm{~nm}$. The unvignetted instrument field of view is $7 \times 7$ arcmin with a pixel scale of 0.125 arcsec. This Letter reports on one of the first scientific results obtained with GTC/OSIRIS after the gamma-ray burst circular of Castro-Tirado et al. (2009) and the observations of SGR $0418+5729$ by Mignani et al. (2009).

We have carried out low-resolution $(R \sim 515$ at $8500 \AA)$ spectroscopy of ULAS1350 with the R500R grism available on GTC/OSIRIS and a slit width of 1 arcsec, projecting onto a full width at half-maximum of seven pixels onto the detector, yielding a 480-1000 nm wavelength coverage and a nominal dispersion of $0.244 \mathrm{~nm} \mathrm{pixel}^{-1}$. The observations were obtained in service mode by the GTC staff on 2009 April 30. Spectra were taken at the parallactic angle. Weather conditions were spectroscopic and seeing around 1.0 arcsec. The total exposure time was $2100 \mathrm{~s}$ in one single exposure. Skyflats and bias frames were observed on 2009 April 4.

All data were reduced under the $\mathrm{IRAF}^{6}$ environment, which includes bias subtraction, and flat-field correction using skyflat images. The individual spectrum was optimally extracted and the Earth sky emission lines were removed with the APALL task by choosing the appropriate aperture width and background intervals. Wavelength calibration (in the air system) was performed with an accuracy of $0.05 \mathrm{~nm}$ using the internal arc lamp lines of Argon and Neon, which were acquired at the end of the night in which ULAS1350 was observed. To correct for the instrumental response, we used twilight skyflat images (taken with the same instrumental configuration

\footnotetext{
6 IRAF is distributed by the National Optical Astronomy Observatories, which are operated by the Association of Universities for Research in Astronomy, Inc., under contract with the National Science Foundation.
} 


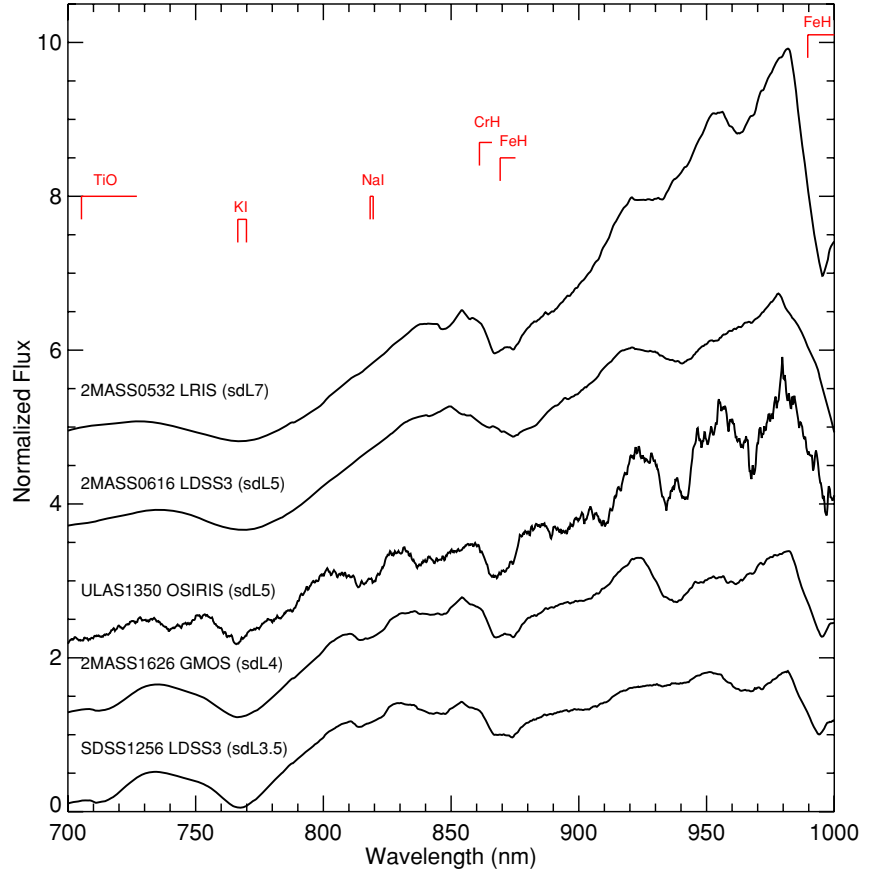

Figure 2. Smoothed $(R \sim 10)$ optical spectrum of ULAS1350 from the GTC. The signal-to-noise ratio of the spectrum after smoothing is of the order of 20 over the $920-925 \mathrm{~nm}$ wavelength range. Overplotted are the optical spectra of the four known L subdwarfs: 2MASS0532 (Keck/LRIS sdL7; Burgasser et al. 2003), 2MASS0616 (Gemini/GMOS; sdL5; Cushing et al. 2009), 2MASS1626 (Gemini/GMOS; sdL4; Burgasser et al. 2007), and SDSS1256 (Magellan/LDSS3; sdL3.5; Burgasser et al. 2009) degraded in wavelength resolution and smoothed in the same manner as ULAS1350. Spectra have been normalized at $8200 \AA$ and are shifted along the $y$-axis by 1.2 for clarity.

(A color version of this figure is available in the online journal.)

as our target) that recorded the solar spectrum. The closest observation of a spectrophotometric standard with the R500R grating was carried out on 2009 April 4 (HZ 44; Oke 1990), the same night as the skyflats. We have used it to confirm that the correction from instrumental response using the twilight flat images is reliable in the wavelength range $700-900 \mathrm{~nm}$, where this star has spectrophotometric information in the IRAF database. Fringing is negligible $(\leqslant 1 \%)$ shortward of $900 \mathrm{~nm}$. The extracted spectrum of ULAS1350 has a signal-to-noise ratio of around 3 at around $920-925 \mathrm{~nm}$. The usable optical (700$1000 \mathrm{~nm}$ ) spectrum of ULAS1350 smoothed with a boxcar of 51 pixels and a signal-to-noise ratio of $\sim 20$ is displayed in Figure 2 and is compared to the spectra (resolution degraded to that of ULAS1350 and similarly smoothed) of the four known $\mathrm{L}$ subdwarfs from the literature.

\section{ANALYSIS \\ 4.1. Photometry}

From the optical and near-infrared photometry alone, ULAS1350 is unlikely to be a solar-metallicity object because it shows discrepant colors compared to late-M and $\mathrm{L}$ dwarfs (Table 1). Its $J-K$ color of $-0.02 \pm 0.16$ mag is typical of $\mathrm{T}$ dwarfs and much bluer than solar-metallicity field L dwarfs discovered by 2MASS and SDSS $(J-K \geqslant 1.2 \mathrm{mag}$; Hawley et al. 2002). Moreover, the $z-J$ and $i-z$ are also much bluer with values of $1.55 \pm 0.07$ and $1.74 \pm 0.11$ mag compared to $\sim 2.5-3.3$ mag and $>2.0$ mag, for mid-L dwarfs with solar composition, respectively.

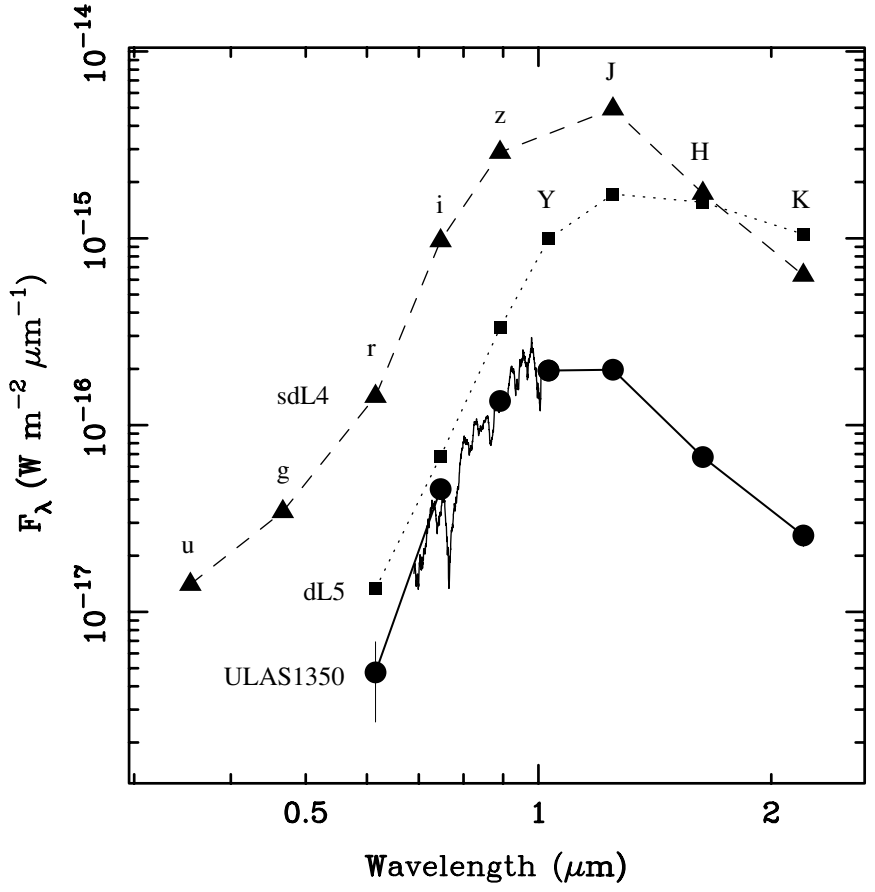

Figure 3. Observed SED of ULAS1350 formed by combining SDSS/UKIDSS photometry and the GTC spectrum (circles and solid line). For comparison purposes, the observed SEDs of the metal-depleted dwarf 2MASS1626 (sdL4; triangles and dashed line; optical and near-infrared photometry from SDSS and Schilbach et al. (2009), respectively) and the solar metallicity dwarf SDSS 1446 (dL5; squares and dotted line; photometry from SDSS and UKIDSS) are also shown. While the slope in the visible wavelengths are rather similar for solar and metal-depleted mid-L dwarfs, the SED appears quite different in the nearinfrared.

Figure 3 shows the optical to near-infrared spectral energy distribution (SED) of ULAS1350 compared to 2MASS1626 (sdL4; Burgasser 2004) and a solar-metallicity L5 dwarf, SDSS J144600.60+002452.0 (Geballe et al. 2002). The SED of ULAS1350 clearly suggests a sub-solar metallicity especially in the near-infrared, as also inferred from Figure 1 and Table 1. To convert observed magnitudes into physical fluxes, we have used the zero point fluxes corresponding to each SDSS and UKIDSS passband defined in Hewett et al. (2006). We have flux calibrated the GTC data by integrating the observed spectrum convolved with the response curves of the $i$ and $z$ filters (Fukugita et al. 1996).

\subsection{Spectral Features}

The GTC spectrum confirms the cool and low-luminosity atmosphere of our candidate, for which we determined sdL5 \pm 1 after comparison to the four known bright L subdwarfs (Figure 2). Indeed, the spectrum of ULAS1350 clearly exhibits a strong pressure-broadened $\mathrm{K}_{\mathrm{I}}$ band at $\sim 770 \mathrm{~nm}$ (Figure 2) as well as a red slope longward of $800 \mathrm{~nm}$, features typical of early- to mid-L field dwarfs. We also detect the hydride bands of $\mathrm{CrH}(\sim 860 \mathrm{~nm})$ and $\mathrm{FeH}(\sim 870 \mathrm{~nm}$ and $\sim 990 \mathrm{~nm}$ ), typically stronger features in the spectra of lower metallicity stars (Mould 1976). From Figure 2, our spectrum shows a decreasing flux at wavelengths below $730 \mathrm{~nm}$, a feature that is also shared by the known sdL3.5-sdL5 objects. It is likely due to absorption by $\mathrm{TiO}$ gas, which is usually stronger in the subdwarfs relative to solar L dwarfs (probably an effect due to inhibited dust formation in low-metallicity atmospheres) (e.g., Burgasser et al. 2003; Reiners \& Basri 2006). While Burgasser 
et al. (2007) proposed a recipe for assigning spectral types to $\mathrm{L}$ subdwarfs by comparing with known solar-metallicity L dwarfs, no set of spectral standards is currently defined due to the few $\mathrm{L}$ subdwarfs known, so we consider our spectral type as tentative.

\subsection{Distance and Tangential Velocity}

We have considered the absolute magnitude versus spectraltype relations given by Cushing et al. (2009) to estimate the distance of ULAS1350 as no L5 subdwarf with known trigonometric parallax exist. Assuming a spectral type of sdL5 and an uncertainty of one subtype, we derive $M_{J}=12.715 \mathrm{mag}$ (12.402-13.028 mag), yielding a mean distance of $111 \mathrm{pc}$ with a possible range from 96 to $128 \mathrm{pc}$. Using the relations for $H$ and $K$, we find a distance $25 \%-30 \%$ larger than for the $J$-band relation. The uncertainty on the distance due to the relations of Cushing et al. (2009) is 13-14 pc, implying that the uncertainty on the distance is dominated by the error on the spectral classification. To account for both uncertainties, we added the errors in quadrature, leading to a spectroscopic distance of $140_{-46}^{+30} \mathrm{pc}$ for ULAS1350.

Combining the proper motion $\left(0.28 \operatorname{arcsec} \mathrm{yr}^{-1}\right)$ and the distance $(140 \mathrm{pc})$ derived above, we derive a tangential velocity of $186_{-61}^{+40} \mathrm{~km} \mathrm{~s}^{-1}$ where the error bars account for the distance uncertainty. On the one hand, these values are $\sim 2-4$ times larger than the mean values of tangential velocity reported for $\mathrm{L}$ dwarfs in the solar neighborhood (Vrba et al. 2004; Faherty et al. 2009). On the other hand, the transverse velocity of ULAS1350 is quite similar to the tangential velocities of the ultracool subdwarfs shown by Schilbach et al. (2009) in their Table 2. This indicates that ULAS1350 likely exhibits halo kinematics. As inferred from Figure 1, state-of-the-art models predict a metallicity between $[\mathrm{M} / \mathrm{H}]=-0.5$ and -1.0 dex for ULAS1350 although current models do not reproduce accurately the color trends of field dwarfs mainly because condensate clouds are not included, resulting in offsets between predicted and observed colors (Figure 1; Burgasser et al. 2009).

\section{DISCUSSION}

ULAS1350 was discovered in a 234 square area common to the SDSS and UKIDSS surveys. Our search was limited to $J$ magnitudes brighter than 18.5. Therefore, we can provide an estimate of the density of L5 subdwarfs in the surveyed area. At the distance of $140 \mathrm{pc}$, the explored volume is approximately $6600 \mathrm{pc}^{-3}$, implying a rough space density of $1.5 \times 10^{-4} \mathrm{sdL} 5 \mathrm{~s}$ per cubic parsecs. The density could, however, be larger as we have six other photometric candidates identified in the same area and we have not yet explored the faint end of the LAS. This discovery could also be fortuitous, yielding an overestimate of the space density. Moreover, ULAS1350 could be a multiple system leading to the sampling of a larger volume. The inferred density is $\sim 20$ times lower than the space density of solar-type field L dwarfs (lower limit of $3.8 \times 10^{-3} \mathrm{pc}^{-3}$; Cruz et al. 2007). It is also 10 times higher than the density of $\mathrm{M}$ subdwarfs for $M_{V}=5-10\left(1-3 \times 10^{-5} \mathrm{pc}^{-3}\right.$; Digby et al. 2003); we note however that our estimation is obtained for fainter and cooler objects, which may have smaller masses. This might indicate that the subdwarf mass function (in a linear scale) is flat or slowly rising toward the Hydrogen burning-mass limit. We remark that this is a tentative suggestion since it is based on the finding of one L5 subdwarf for which distance is not known precisely. If we extrapolate our findings to the final area imaged by the LAS with SDSS photometry $\left(\sim 3000 \mathrm{deg}^{2}\right)$, we would expect roughly 13 mid-L subdwarfs. This tentative number shows that our search may open new prospects of designing an accurate spectral classification in the $\mathrm{L}$ dwarf regime including metallicity as a variable parameter (Kirkpatrick 2005). With this aim, we have conducted a cross-match of UKIDSS DR6 and SDSS DR7 following a Virtual Observatory methodology whose results will be presented in a forthcoming Letter.

N.L. was funded by the Ramón y Cajal fellowship number 08303-01-02. This work was partially funded by Spanish Ministry of Science grant number AYA2007-67458 and the ConsoliderIngenio 2010 Program grant CSD2006-00070: "First Science with the GTC (http://www.iac.es/consolider-ingenio-gtc)." N.L. thanks numerous discussions on subdwarfs with Richard Jameson during his post-doc in Leicester. We thank the GTC staff for their help as well as Adam Burgasser and Mike Cushing for providing the optical spectra of the known L subdwarfs used in this Letter. We thank the anonymous referee for his/her prompt and useful report.

Based on observations made with the Gran Telescopio Canarias (GTC), installed in the Spanish Observatorio del Roque de los Muchachos of the Instituto de Astrofísica de Canarias, in the island of La Palma.

The United Kingdom Infrared Telescope is operated by the Joint Astronomy Centre on behalf of the U.K. Science Technology and Facility Council.

The SDSS is managed by the Astrophysical Research Consortium for the Participating Institutions. The Participating Institutions are the American Museum of Natural History, Astrophysical Institute Potsdam, University of Basel, University of Cambridge, Case Western Reserve University, University of Chicago, Drexel University, Fermilab, the Institute for Advanced Study, the Japan Participation Group, Johns Hopkins University, the Joint Institute for Nuclear Astrophysics, the Kavli Institute for Particle Astrophysics and Cosmology, the Korean Scientist Group, the Chinese Academy of Sciences (LAMOST), Los Alamos National Laboratory, the Max-PlanckInstitute for Astronomy (MPIA), the Max-Planck-Institute for Astrophysics (MPA), New Mexico State University, Ohio State University, University of Pittsburgh, University of Portsmouth, Princeton University, the United States Naval Observatory, and the University of Washington.

\section{REFERENCES}

Abazajian, K., et al. 2005, AJ, 129, 1755

Baraffe, I., Chabrier, G., Allard, F., \& Hauschildt, P. H. 1997, A\&A, 327, 1054 Burgasser, A. J. 2004, ApJ, 614, L73

Burgasser, A. J., Cruz, K. L., \& Kirkpatrick, J. D. 2007, ApJ, 657, 494

Burgasser, A. J., Geballe, T. R., Leggett, S. K., Kirkpatrick, J. D., \& Golimowski, D. A. 2006, ApJ, 637, 1067

Burgasser, A. J., Witte, S., Helling, C., Sanderson, R. E., Bochanski, J. J., \& Hauschildt, P. H. 2009, ApJ, 697, 148

Burgasser, A. J., et al. 2003, ApJ, 592, 1186

Casali, M., et al. 2007, A\&A, 467, 777

Castro-Tirado, A. J., et al. 2009, GRB Coordinates Network, 9655, 1

Cepa, J., et al. 2000, Proc. SPIE, 4008, 623

Cruz, K. L., et al. 2007, AJ, 133, 439

Cushing, M. C., et al. 2009, ApJ, 696, 986

Cutri, R. M., et al. 2003, 2MASS All Sky Catalog of Point Sources, 2246

Digby, A. P., Hambly, N. C., Cooke, J. A., Reid, I. N., \& Cannon, R. D. 2003, MNRAS, 344, 583

Dye, S., et al. 2006, MNRAS, 372, 1227

Faherty, J. K., Burgasser, A. J., Cruz, K. L., Shara, M. M., Walter, F. M., \& Gelino, C. R. 2009, AJ, 137, 1

Fukugita, M., Ichikawa, T., Gunn, J. E., Doi, M., Shimasaku, K., \& Schneider, D. P. 1996, AJ, 111, 1748 
Geballe, T. R., et al. 2002, ApJ, 564, 466

Hambly, N. C., et al. 2008, MNRAS, 384, 637

Hawley, S. L., et al. 2002, AJ, 123, 3409

Hewett, P. C., Warren, S. J., Leggett, S. K., \& Hodgkin, S. T. 2006, MNRAS, 367,454

Jao, W.-C., Henry, T. J., Beaulieu, T. D., \& Subasavage, J. P. 2008, AJ, 136, 840

Kirkpatrick, J. D. 2005, ARA\&A, 43, 195

Kirkpatrick, J. D., et al. 1999, ApJ, 519, 802

Lawrence, A., et al. 2007, MNRAS, 379, 1599

Lépine, S., \& Scholz, R.-D. 2008, ApJ, 681, L33

Linsky, J. L. 1969, ApJ, 156, 989

Lodieu, N., Leggett, S. K., Bergeron, P., \& Nitta, A. 2009a, ApJ, 692, 1506

Lodieu, N., Zapatero Osorio, M. R., \& Martín, E. L. 2009b, A\&A, 499, 729

Luyten, W. J. 1980, NLTT Catalogue, Vol. 3: 0 deg. to -10 deg (Minneapolis, MN: Univ. Minnesota)

Martín, E. L., Delfosse, X., Basri, G., Goldman, B., Forveille, T., \& Zapatero Osorio, M. R. 1999, AJ, 118, 2466
Mignani, R. P., et al. 2009, ATel, 2044, 1

Miller, G. E., \& Scalo, J. M. 1979, ApJS, 41, 513

Mould, J. L. 1976, ApJ, 207, 535

Oke, J. B. 1990, AJ, 99, 1621

Reiners, A., \& Basri, G. 2006, AJ, 131, 1806

Riaz, B., Gizis, J. E., \& Samaddar, D. 2008, ApJ, 672, 1153

Salpeter, E. E. 1955, ApJ, 121, 161

Scalo, J. M. 1986, Fundam. Cosm. Phys., 11, 1

Schilbach, E., Röser, S., \& Scholz, R. 2009, A\&A, 493, L27

Scholz, R.-D., Lodieu, N., \& McCaughrean, M. J. 2004, A\&A, 428, L25

Sivarani, T., Lépine, S., Kembhavi, A. K., \& Gupchup, J. 2009, ApJ, 694, L140

Skrutskie, M. F., et al. 2006, AJ, 131, 1163

Tokunaga, A. T., Simons, D. A., \& Vacca, W. D. 2002, PASP, 114, 180

Vrba, F. J., et al. 2004, AJ, 127, 2948

Warren, S. J., et al. 2007, arXiv:astro-ph/0703037

West, A. A., et al. 2008, AJ, 135, 785

York, D. G., et al. 2000, AJ, 120, 1579 